\title{
Programmed Vehicle Accident Detection using GSM and GPS Modem and Prevention using Alcohol Sensor
}

\author{
Jayesh Choudhary $^{1}$, Manish Kumar Meena ${ }^{2}$, Saroj Kumar Sah ${ }^{3}$, Mrs. Alka Rani ${ }^{4}$ \\ UG, Poornima Group of Institutions, Jaipur, India ${ }^{1,2,3}$ \\ Asst. Professor EC Department Poornima Institute of Engineering and Technology, Jaipur, India ${ }^{4}$
}

\begin{abstract}
The Rapid development of innovation and framework has made our lives less demanding. The coming of innovation has additionally expanded the movement perils and the street mischances happen oftentimes which causes gigantic death toll and property as a result of the poor crisis offices. Our venture will give an ideal answer for this downside. An accelerometer can be utilized as a part of an auto caution application so that unsafe driving can be recognized. It can be utilized as a crash then again rollover identifier of the vehicle amid and after a crash. With signs from an accelerometer, an extreme mischance can be perceived. As indicated by this venture when a vehicle meets with a mishap instantly Vibration sensor will identify the flag or if an auto moves over, and Micro electro mechanical framework (MEMS) sensor will identifies the flag and sends it to ARM controller. Microcontroller sends the ready message through the GSM MODEM including the area to police control room or a safeguard group. So the police can instantly follow the area through the GPS MODEM, in the wake of getting the data. At that point subsequent to acclimating the area essential move will be made. On the off chance that the individual meets with a little mishap or if there is no genuine risk to anyone`s life, then the ready message can be ended by the driver by a switch gave so as to abstain from squandering the profitable time of the medicinal safeguard group. This paper is successful in recognizing the mishap absolutely by way of doing thing for both vibration sensor and Micro electro Mechanical framework (MEMS) or accelerometer. As there is a field of reference for development and as an age execution we can include a remote gps system for catching the location which will aid in giving victim help. Here we have discussed on alcohol sensor which will indicate the person can drive the car or not and will allow the car to get started or stop. This will increase the safety parameters of person.
\end{abstract}

Keywords: GSM, GPS, Vibration Sensor, ARM Controller, MEMS.

\section{INTRODUCTION}

Security in travel is primary approach for aside one. This Project describes a raw material of effective dissuade program that can inspect an automotive / medium / car precondition in traveling. This business is designed to acknowledge virtually a force majeure that is reach a process to the person in the street members of the spent persons. This long row to hoe uses a Piezo-electric sensor which boot regard the candid vibration when an accident is occurred. This sends a signal to microcontroller.

This Project presents an automatic vehicle accident detection system for GPS and GSM modems and prevention by detecting the alcohol meter of a person and will disable the vehicle. The system can be interconnected by en masse of the car dissuade system and sharp the person of the house on his mobile phone. This detection and messaging system is composed of a GPS receiver, Microcontroller and a GSM Modem. GPS Receiver gets the location information from satellites in consist of latitude and longitude.

The Microcontroller processes this information and this able information is sent to the user/owner by the agency of GSM modem A GSM modem is interfaced to the MCU.
The GSM modem sends an SMS to the predefined mobile number and informs about this accident. This enable it to detect the accident situations and it can immediately alerts the police/ambulance service mutually the location of accident. This works for the detection of accident and for the prevention purpose there is alcohol sensor in the vehicle and will sense the driver is drunk enough and won't be able to drive the car then the car system will be disable and won't start till someone else is driving the vehicle.[1] The project is built during the AT89S52 micro controller from Atmel. This micro controller provides all the functionality of the SMS alert system. It furthermore takes service of filtering of the signals at the inputs.

The freshness of this project is, not unattended alerting the neighbors by its wizard, but by the same token it sends SMS to four mobile numbers. This numbers can be driven at any time by the user using a $3 \times 4$ key pad. These numbers are brought together in EEPROM.

This project uses regulated $5 \mathrm{~V}, 750 \mathrm{~mA}$ power supply. 7805 three terminal voltage regulator is used for voltage regulation. Bridge type full wave rectifier is hand me down 
to rectify the ac output of secondary of $230 / 12 \mathrm{~V}$ step down transformer.

\section{LITERATURE SURVEY}

At present criteria, we cannot detect to what place the accident has occurred and from this point forward no information thick to it, leading to the death of an individual. The research work is going on for tracking the position of the vehicle someday in dark clumsy areas to what place there is no network for receiving the signals and before the accident can take place as drunk and drive is one of the major reasons of accidents so this research paper can detect this problem and may provide some sort of solution to it. In this project GPS is used for tracking the position of the vehicle, GSM is used for sending the message and the ARM controller is used for saving the mobile number in the EEPROM and alcohol sensor is used to detect the driver is drunk enough or not to drive and sends the message to it when an accident has been detected. [2]

Hence with this project implementation we can detect the position of the vehicle where the accident has occurred in case we can provide the first aid as early as possible.

\section{HARDWARE DESCRIPTION}

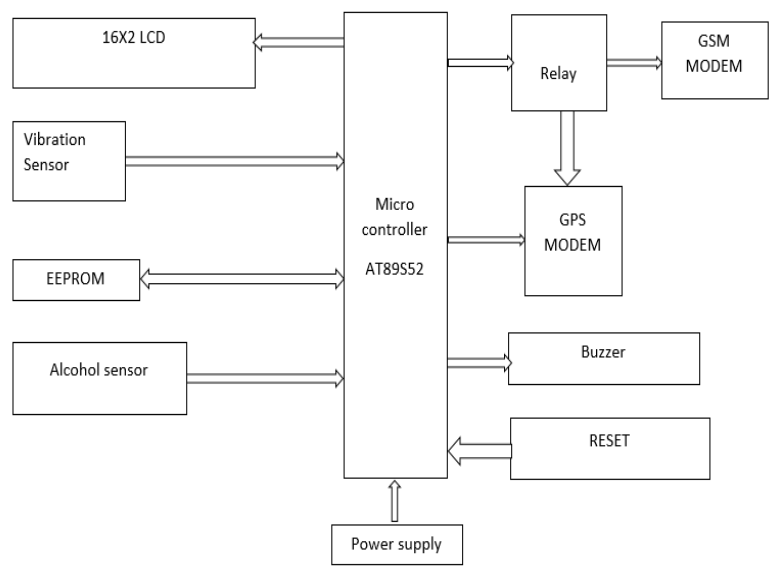

Fig. block diagram

The system incorporates 89S52 microcontroller, Alcohol sensor, vibration sensor, Global Positioning System (GPS), Global System for Communication (GSM). The vibration sensor works on the piezoelectric property of the crystals and produces an electric signal as it senses vibrations of the unit and gives the signal as input to the microcontroller output given to relays. A relay is an electrically operated switch. It is used where electrical isolation is to be provided between controlled and controlling system. In running condition the 1 st relay $i$ it ensures that the vehicle runs under the normal working condition. As shortly as an accident is detected, that is if the sensor signal values deviate from the specific limits previously microcontroller engine will stop working, thus stopping the car. Another relay is connected to the air-bag. And it is in normally open state. The air compressor is activated and airbag blows as shortly as the controller signal goes high.at every moment the current location of the microcontroller with the accident location details. MAX232 IC converts signals from anRS232 serial hard to left to signals sufficient for act with regard to in TTL germane digital purport circuits.it synchronizes baud rates of microcontroller and GSM modem. [3][4]

\section{A. $16 * 2$ LED}

$16 \mathrm{X}$ a pair of LCD is employed to indicate the manual and standing of the output. HD44780U is employed within the project. The HD44780U dot-matrix liquid show controller and driver LSI displays alphabetical, Japanese kana characters, and symbols. It bouncecel be designed to charge a dot-matrix click crystal show below the management of a 4- or 8-bit silicon chip. Since all the functions like show RAM, character generator, and liquid driver, needed for driving a dot-matrix liquid show ar internally provided on such chip, a lowest system bouncecel be interfaced by all of this controller/driver. a success HD44780U will show up to at least one 8-character orbit or 28 -character lines. The HD44780U has pin perform compatibility reciprocally the HD44780S that permits the junkie to simply replace AN LCD-II with AN HD44780U. The HD44780U character motor fixed storage is extended to provoke $2085 \mathrm{X} 8$ peyote character fonts and thirty two $5 \times 10$ dot character fonts for an accumulation of 240 completely different character fonts.

\section{B. Vibration Sensor}

Despite the advances created in vibration observance and analysis instrumentality, the choice of sensors and also the approach they're mounted on a machine stay important factors in determinative the success of any computer program. Cash saved by putting in inferior sensors isn't a prudent investment since the data provided concerning the machine of interest typically isn't correct or reliable. Poor quality sensors will simply provide dishonorable information or, in some cases, cause an important machine condition to be fully unnoticed. consistent with this project once a vehicle meets with AN accident directly Vibration device can sight the signal or if a automobile rolls over, and Piezo device can detects the signal and sends it to ARM controller Microcontroller sends the alert message through the GSM electronic equipment together with the situation to police room or a rescue team. Therefore the police will directly trace the situation through the GPS electronic equipment, when receiving the data.

\section{EEPROM}

24C04 EEPROM is employed during this project. This EEPROM stores the mobile numbers entered by the user for receiving accident alert SMS. The info keep within the EEPROM can retain even the ability is off for longstanding.

\section{Alcohal sensor}

An alcohol device detects the attentiveness of alcohol gas within the air and an analog voltage is an output reading. 
The device will activate at temperatures starting from -10 to $50^{\circ} \mathrm{C}$ with an influence provide is a smaller amount than a hundred and fifty $\mathrm{Ma}$ to $5 \mathrm{~V}$. The sensing vary is from zero. $04 \mathrm{mg} / \mathrm{L}$ to four $\mathrm{mg} / \mathrm{L}$, that is appropriate for device. The most purpose behind this project is "Drunk driving detection". Now a days, several accidents are happening thanks to the alcohol consumption of the motive force or the one that is driving the vehicle so Drunk driving could be a major reason of accidents in the majority countries everywhere the planet once drunk someone when than set in automobile then begin and it determine an a automobile are going to be not beginning it basic benefits of alcohol device.

\section{E. Microcontrollers AT89S52}

The 89S52 has 4 different ports, each one having 8 Input/output lines providing a total of $32 \mathrm{I} / \mathrm{O}$ lines. Those ports can be used to output DATA and orders do other devices, or to read the state of a sensor, or a switch. Most of the ports of the 89S52 have 'dual function' meaning that they can be used for two different functions. The first one is to perform input/output operations and the second one is used to implement special features of the microcontroller like counting external pulses, interrupting the execution of the program according to external events, performing serial data transfer or connecting the chip to a computer to update the software. Each port has 8 pins, and will be treated from the software point of view as an 8-bit variable called 'register', each bit being connected to a different Input/output pin. Here are two different memory types: RAM and EEPROM. Shortly, RAM is used to store variable during program execution, while the EEPROM memory is used to store the program itself, that's why it is often referred to as the 'program memory'. It is clear that the CPU (Central Processing Unit) is the heart of the micro controllers. It is the CPU that will Read the program from the FLASH memory and execute it by interacting with the different peripheralsDiagram below shows the pin configuration of the 89S52, where the function of each pin is written next to it, and, if it exists, the dual function is written between brackets. Note that the pins that have dual functions can still be used normally as an input/output pin. Unless the program uses their dual functions, all the $32 \mathrm{I} / \mathrm{O}$ pins of the microcontroller are configured as input/output pins.

\section{F. Relay}

A relay is an electromagnetic switch operated by a relatively small electric current that can turn on or off a much larger electric current. The heart of a relay is an electromagnet (a coil of wire that becomes a temporary magnet when electricity flows through it). [5]

\section{G. GSM Modem}

A GSM electronic equipment is a device which may be either an itinerant or an electronic equipment device which may be wont to build a laptop or the other processor communicate over a network. A GSM electronic equipment needs a SIM card to be operated and operates over a network vary signed by the network operator. It will be connected to a laptop through serial, USB or Bluetooth affiliation. A GSM electronic equipment can even be a typical GSM itinerant with the acceptable cable and package driver to attach to an interface or USB port on your laptop. GSM electronic equipment is sometimes preferred to a GSM itinerant. The GSM electronic equipment has wide selection of applications in group action terminals, offer chain management, security applications, weather stations and GPRS mode remote knowledge logging's could be a mobile communication modem; it's stands for world system for mobile communication (GSM). The thought of GSM was developed at Bell Laboratories in 1970. It's wide used mobile communication system within the world. GSM is Associate in Nursing open and digital cellular technology used for transmission mobile voice and knowledge services operates at the $850 \mathrm{MHz}, 900 \mathrm{MHz}, 1800 \mathrm{MHz}$ and $1900 \mathrm{MHz}$ frequency bands.GSM system was developed as a digital system exploitation time division multiple access (TDMA) technique for communication purpose.

A GSM digitizes and reduces the information, then sends it down through a channel with 2 totally different streams of consumer knowledge, every in its own specific slot. The digital system has a capability to hold sixty four kbps to one hundred twenty Mbps of information rates. There are numerous cell sizes during a GSM system like macro, micro, Pico and umbrella cells. Every cell varies as per the implementation domain. There are 5 totally different cell sizes during a GSM network macro, micro, Pico and umbrella cells. The coverage space of every cell varies per the implementation atmosphere

\section{H. GPS Modem}

Global Positioning System (GPS) is a worldwide radionavigation system shaped from the constellation of twenty four satellites and their ground stations. The worldwide Positioning System is principally funded and controlled by the U.S Department of Defence (DOD). The system was abs initio designed for the operation of U. S. military. But today, there also are several civil users of GPS across the entire world. The civil user's square measure allowed to use the quality Positioning Service with none reasonably charge or restrictions. World Positioning System pursuit may be a technique of understanding specifically wherever one thing is. A GPS pursuit system, as an example, could also be placed in an exceedingly vehicle, on a mobile phone, or on special GPS devices, which may either be a hard and fast or transportable unit. GPS works by providing info on precise location. It can even track the movement of a vehicle or person. A GPS pursuit system uses the worldwide Navigation Satellite System (GNSS) network. This network incorporates a variety of satellites that use microwave signals that square measure transmitted to GPS devices to allow info on location, vehicle speed, time and direction. So, a GPS pursuit system will doubtless provide each time period and historic navigation knowledge on any reasonably journey. 
An active GPS chase system is additionally referred to as a period system as this methodology mechanically sends the data on the GPS system to a central chase portal or system in period because it happens. This is often additionally a helpful approach of observance the behaviour of staff as they perform their work and of streamlining internal processes and procedures for delivery fleets. Period chase is additionally significantly helpful from a security perspective because it permits vehicle homeowners to pinpoint the precise location of a vehicle at any given time. And, the GPS chase system within the vehicle could then be ready to facilitate police investigation out wherever the vehicle was taken to if it absolutely was taken.

\section{Buzzer}

Buzzers are offered in an exceedingly style of designs. Magnetic buzzers emit audio via a coil, whereas mechanical device buzzers might use a bell in their style. Mechanical buzzers don't operate with electricity. Instead, they think about a tightly wound spring. Hand buzzers and alarm clocks that square measure manually wound square measure mechanical in style. Piezo buzzers will emit a range of beeps and buzzes and square measure usually used on automotive doors and to point pc errors. Buzzers square measure used to be used on microwave ovens and standard ovens moreover. temporal order devices rely upon buzzers, as do alarms for seat belts and dashboard warning lights. Buzzers also are used throughout recreational activities. Most game shows need players to hit a buzzer after they square measure able to answer a matter or need to signal the host. at school and sport games, buzzers sound once the clock runs out of your time.

\section{J. Reset}

Reset approach circuit is secondhand to reset the microcontroller at any stage of labor. This section besides includes of automotive vehicle away with on reset. If the reset relieve is ironed, the microcontroller restarts and therefore the work can begin from the start. This circuit is connected to ninth pin of microcontroller.

\section{K. Power provide}

A power provide is Associate in Nursing device that provides electrical energy to Associate in Nursing electrical load. the first perform of an influence provide is to convert one variety of current to a different and, as a result, power provides square measure generally noted as power converters. Some power provides square measure distinct, complete devices, whereas others square measure engineered into larger devices together with their masses.

\section{WORKING PRINCIPLE}

A. Detection Phase

Our paper describes practically the AUTOMATIC VEHICLE ACCIDENT DETECTION AND MESSAGING SYSTEM using GPS and GSM technologies. We are for AT89C52 microcontroller in our project. When the system is switched on, LED will be ON indicating that power is supplied to the circuit. When the IR sensors that we are for in our project sense whole barrier, they send interrupt to microcontroller.

The GPS receives the location of the vehicle that met by all of an accident and gives the information back. This information will be sent to a mobile number at the hand of a message. This message will be received per GSM modem disclose in the circuit. The message will give the information of longitude and latitude values. Using these values the position of the vehicle can be estimated. Modem performs modulation during transmission and performs demodulation during reception. GSM modem is bringing to mind to mobile phone without any display, keypad, and speakers. It can send and receive messages and calls. The announcement will be sent to MAX232 IC through RS232 cable.MAX232 synchronizes the baud rates of modem and microcontroller.

It also converts RS 232 voltage levels to TTL voltage levels and vice versa. In RS 232, Logic 0 is represented in between 3 volts to 25 volts, Logic 1 is represented in between -3 to -25 volts. In TTL logic 0 is represented as nothing volts heart 1 is represented as 5 volts. Microcontroller supports TTL voltage levels as a substitute than RS 232 voltage levels. The received disclosure is supposing to the microcontroller. Correspondingly it gives an acknowledgement in the art an element of an SMS to the mobile phone. LCD hand me down in the travel displays the nod of messages. The microcontroller is interfaced to GPS and GSM module for a multiplexer, to what place these devices are activated by the agency of select lines internally off the rack in the multiplexer.

Multiplexer is interfaced to microcontroller by transmit and engage pin. LCD is interfaced to any ports of micro controller, it is hand me down to display the current status of the GPS and GSM modules, whether data is been reading from GPS or mail to GSM Most digital interpretation circuits and processors require a 5 volt capacity supply.

To consider these parts we prefer to spawn a businesslike 5 volt source. Usually you run with an unregulated power to make a 5 volt power lend, we manage a LM7805 voltage regulator IC (Integrated Circuit). [6]

\section{B. Prevention Phase}

There is Alcohol sensor connected with the micro controller which will be placed in the car steering. As soon as driver wants to start the vehicle and if he is drunk enough then sensor will detect the alcohol from it. This will give signal to microcontroller and the pin from controller will stop the power supply of the vehicle. So the vehicle won't start until new person sits on the driver seat and drive the car. 


\section{ADVANTAGES \& APPLICATIONS}

A. Advantages

a) Easy to detect the exact location of the vehicle.

b) It provides security to the vehicle in indeed reasonable cost.

c) It saves the victim from being alone and suffering after the accident and gets help.

d) As prevention measures with the alcohol sensor it will limit the number of accidents.

B. Applications

a) Security and remote monitoring of vehicles especially during military operations

b) Trucks, school vehicle and cars accident on highways detection

c) Used in automotive and transport vehicles from lighter vehicles like cars, to heavier automotive like ships and aero planes.

d) Family driver who is drunk after party won't be able to drive the car so it can be used in personal cars.

\section{CONCLUSION}

In this function, we have nicely designed vehicle circumstances beyond one control detection and tracking program by via GSM and GPS. When accident occurs, it senses by PUSH ON SWITCHES. The coordinates of location of circumstances beyond one control obtained by GPS, are sent using GSM network to user defined mobile number. It is the facts of life that implementation of position will overcome cost of vehicle anyhow it is better to have small number percent safety preferably than having no percent of safety. The expected approach is verified to be intensely beneficial for the automotive industry. The proposed system can further be used for traffic estimate and accidents survey in the country by health department mutually slight modification.

\section{FUTURRE SCOPE}

This program can be interfaced mutually vehicle airbag system that stop vehicle occupant from striking interior objects one as the steering wheel or window. This can further be bettered by locking all the brakes automatically in situation of accident. Mostly in accidents, it becomes serious as the drivers lose act and avoid to stop the vehicle. In a well-known cases, the vibration sensor will be triggered seeing of the vibrations confirmed and furthermore processed every processor. The processor be directed be linked to the devices which can lock the brakes when triggered. With this modification, we can prevent the power and can halt the impact of the accident.

\section{ACKNOWLEDGEMENT}

We wish to express immense pleasure and sincere thanks to Mrs. Alka Raniand his staff for their valuable guidance and support for project, Poornima group of institutions, sitapura, Jaipur. This work is simply reflection of their thoughts, ideas and concepts. Working under their guidance was a privilege and an excellent learning experience that will cherish us for a long time.

I would like to express my gratitude to project coordinator Mr. Ankur Sharia, he was responsible to supervise and monitor my progress of this project. My Guide has been patiently monitoring my progress and guided me in the right direction and offering his encouragement to me. I am grateful to my guide for always being my guidance and advisor throughout the duration of the project. Otherwise, this project has not been possible. I have learnt a lot under her guidance, be it practically or theoretically.

I also thank my family as well as college department that always stands by me no matter what happens. Their full support and encouragement were such a boost for my capabilities and confidence to undergo this period.

Last but certainly not least, I also want to thank all my friends for their invaluable assistances towards this project. I must admit here that it was impossible for me to complete my project without the supports of them that I mentioned above.

\section{REFERENCES}

[1] Ashish Kushwaha, Gaurav Katiyar, \&Harshita Katiyar, Hemant Yadav, Saxena 'GPS And GSM Based Accident Alarm System' ;National Student Conference On "Advances in Electrical \& Information Communication Technology"AEICT-2014

[2] C.Prabha 1, R.Sunitha 2, R.Anitha ,"Automatic Vehicle Accident Detection and Messaging System Using GSM and GPS Modem",DOI: 10.15662/ijareeie.2014.0307062.

[3] Mr.Dinesh Kumar HSDK, Shreya Gupta, Sumeet Kumar, Sonali Srivastava“Accident Detection and Reporting System Using GPS and GSM Module.," JETIR (ISSN-2349-5162), May 2015, Volume 2 , Issue 5.

[4] https://www.wikiwand.com/en/Transducer.

[5] http://www.explainthatstuff.com/howrelayswork.html.

[6] Sri Krishna Chaitanya Varma, Poornesh, Tarun Varma, Harsha," Automatic Vehicle Accident Detection And Messaging System Using GPS and GSM Modems ", International Journal of Scientific \& Engineering Research, Volume 4, Issue 8, August-2013 1937. 